\title{
Localized Hepatic Tuberculosis with Imaging Changes Caused by the Progression of Tuberculosis
}

\author{
Tomohiro Iwasaki ${ }^{1}$, Aiko Nagashima ${ }^{1}$, Hideki Nakatsuka ${ }^{2}$ and Norio Ogata ${ }^{1}$
}

\begin{abstract}
Localized hepatic tuberculosis (LHTB) is difficult to diagnose preoperatively, and most cases of LHTB are diagnosed based on pathological findings. A relationship between imaging features and the pathological stage of hepatic tuberculosis (TB) has recently been reported, which could aid in the diagnosis of hepatic TB. We herein present a case study of a patient with LHTB diagnosed postoperatively who demonstrated imaging changes due to the progression of TB. An awareness of the presence of LHTB might have permitted a preoperative diagnosis. This is the first report of an LHTB patient who exhibited imaging changes during the course of the disease.
\end{abstract}

Key words: localized hepatic tuberculosis, EOB-MRI, imaging change

(Intern Med 55: 613-616, 2016)

(DOI: 10.2169/internalmedicine.55.5607)

\section{Introduction}

Although the incidence of tuberculosis (TB) has decreased in the past decades, the recent increase in the incidence of TB is associated with the acquired immunodeficiency syndrome (AIDS) epidemic, increases in intravenous drug abuse and a rise in the number of immunocompromised patients $(1,2)$. TB infection can affect any organ. Miliary hepatic TB is common (3), whereas localized hepatic TB (LHTB), which is defined in this article as tuberculous hepatic involvement without evidence of extrahepatic $\mathrm{TB}$, is rare (4).

LHTB involves no specific symptoms, laboratory abnormalities or imaging features; therefore, it is difficult to diagnose this condition preoperatively (5). However, because anti-TB therapy (ATT) is usually effective in cases of hepatic TB (6), unnecessary surgery can be avoided if LHTB is diagnosed early.

Imaging findings change with the pathological stage of hepatic TB (7-9). Hence, a more thorough understanding of the relationship between the imaging findings and the pathologic stage of TB may help in establishing the correct diagnosis in patients with hepatic TB (8).

In this study, we describe a case of LHTB in which a central, non-enhancing area on gadolinium-ethoxybenzyl diethylenetriamine pentaacetic acid-enhanced magnetic resonance imaging (EOB-MRI) appeared over a short period, which may have reflected the development of caseous necrosis. To the best of our knowledge, this is the first report of an LHTB patient showing imaging changes caused by the progression of $\mathrm{TB}$ during the course of the disease.

\section{Case Report}

A hypertensive 75-year-old man with chronic hepatitis $\mathrm{C}$ $(\mathrm{CHC})$ was treated with pegylated-interferon and ribavirin and achieved a sustained virological response (SVR) in 2009. Subsequently, he continued to visit our hospital regularly. No hepatic masses were observed on a computed tomography (CT) scan performed in January 2011. In January 2012, an abdominal CT scan revealed a low-density lesion measuring $1.5 \mathrm{~cm}$ in segment $5 / 6$ that displayed arterial enhancement without distinct washout in the portal venous phase (Fig. 1a-c). In February 2012, EOB-MRI indicated that the size of this mass lesion was the same as that observed on the CT scan. The lesion was hypointense on T1weighted images and hyperintense on T2-weighted images. The perfusion of the lesion, as assessed on EOB-MRI, was similar to that observed on the CT scan (Fig. 1d, e). How-

${ }^{1}$ Department of Internal Medicine, Tsubame Rosai Hospital, Japan and ${ }^{2}$ Department of Surgery, Tsubame Rosai Hospital, Japan Received for publication April 17, 2015; Accepted for publication June 14, 2015

Correspondence to Dr. Tomohiro Iwasaki, iwattch_1125@yahoo.co.jp 

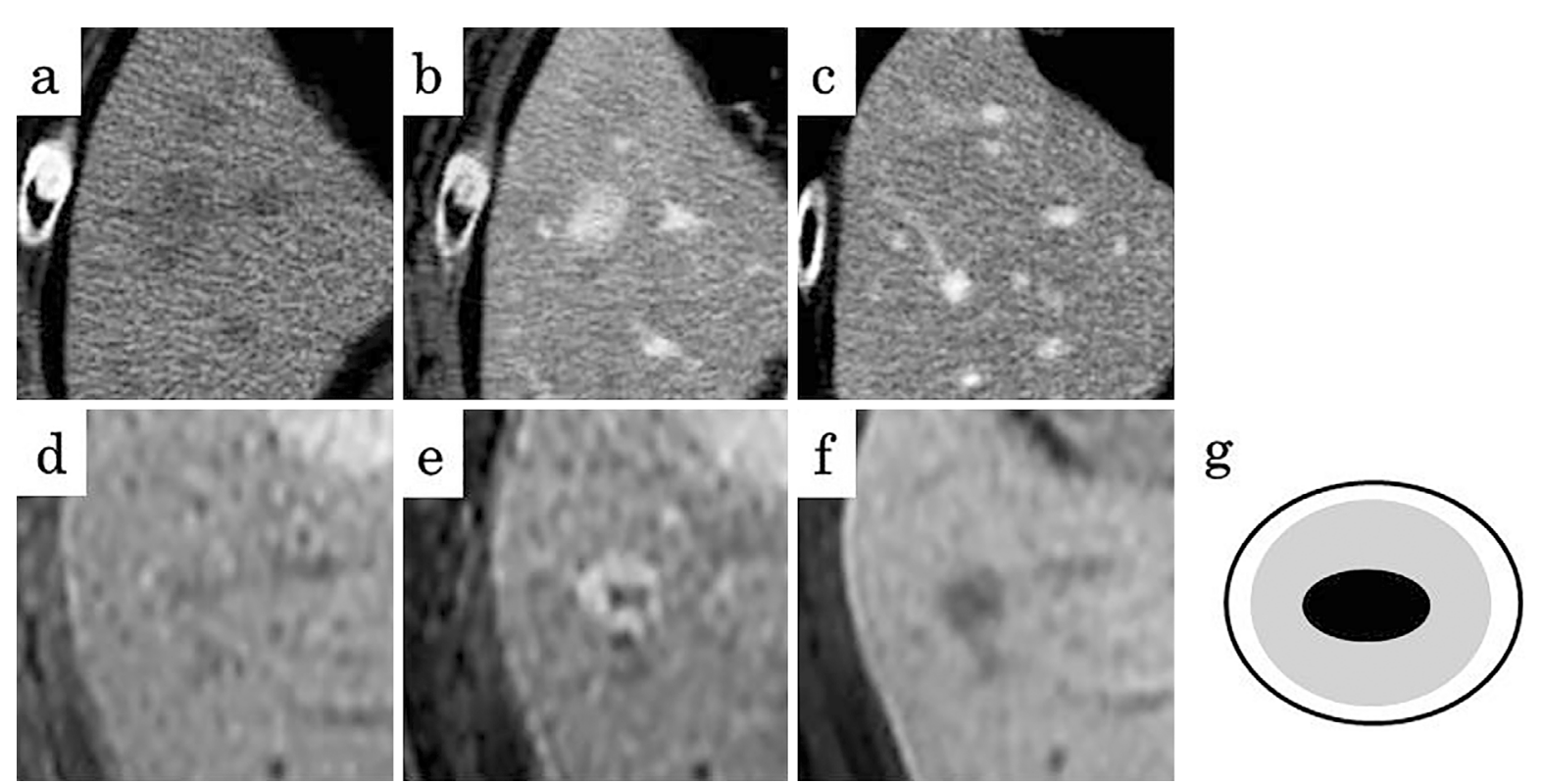

Figure 1. Contrast-enhanced abdominal CT scans obtained in January 2012 and EOB-MRI scans obtained in February 2012. (a) Pre-contrast CT, (b) arterial phase, (c) portal venous phase. A lowdensity lesion measuring $1.5 \mathrm{~cm}$ with arterial enhancement without apparent washout in the portal venous phase was detected in segment 5/6. (d) Pre-contrast MRI, (e) arterial phase, (f) hepatobiliary phase. The lesion was hypointense on pre-contrast MRI and showed arterial enhancement without washout in the portal venous phase. A small non-enhancing area appeared inside the lesion. The hepatic mass lesion displayed low intensity in the hepatobiliary phase and was slightly smaller than the area of arterial enhancement. (g) Schematic figure of the findings noted on EOB-MRI. The black zone shows the non-enhancing area in all vascular phases. The gray zone displays the area of arterial enhancement with hypointensity in the hepatobiliary phase. The white zone demonstrates the area of arterial enhancement with iso-intensity in the hepatobiliary phase.

ever, a small non-enhancing area appeared in the center of this lesion in all vascular phases. In the hepatobiliary phase, the lesion had low signal intensity (Fig. 1f) and was slightly smaller than the arterially enhancing area. The lesion was also hyperintense on diffusion-weighted (DW) images, and the hepatic mass displayed homogeneous hypoechoic echogenicity on abdominal ultrasonography.

The patient was asymptomatic, and a chest X-ray was normal. Laboratory testing revealed only mild elevation in the alkaline phosphatase (ALP) level (Table). The $\alpha$ fetoprotein (AFP) and prothrombin values induced by vitamin K absence-II (PIVKA-II) were within the normal limits. We diagnosed the lesion as hepatocellular carcinoma (HCC), and partial hepatectomy was performed in March 2012. A histological examination of the resected hepatic mass lesion revealed caseous necrosis surrounded by granulomas, thereby establishing the diagnosis of TB (Fig. 2a, b). Acidfast bodies were not identified. The patient had had no contact with any other TB patients. CT scans of the chest and abdomen, endoscopic gastroduodenoscopy and colonoscopy did not show any extrahepatic tuberculous involvement. Based on these results, we diagnosed the hepatic mass to be LHTB. ATT was not performed because active TB involvement was not detected after surgery. No tuberculous lesions have been observed since the operation.

\section{Discussion}

Hepatic TB is classified into three forms: miliary, diffuse and localized hepatic (10-13). The miliary form is common, whereas the localized hepatic form is rare. This case was categorized as the localized hepatic form of the disease, and there was only one hepatic tuberculoma, which is rare.

It is difficult to accurately diagnose LHTB because it is a rare disease without specific symptoms, laboratory abnormalities or imaging features. Oliva et al. reported 23 cases of LHTB; in 20 of the 23 patients, the LHTB lesions were preoperatively misdiagnosed as carcinoma of the liver or a liver abscess (14). Common symptoms of hepatic TB include fever, weight loss and abdominal pain, which are nonspecific $(6,10,15,16)$. A common laboratory abnormality is an elevated ALP level $(5,6,14,16,17)$. Imaging studies show various non-specific features. In most cases of LHTB, a histological examination is required to make a correct diagnosis $(5,6)$. In the current case, the patient was asymptomatic, and the only abnormal laboratory result was slight elevation of ALP. Based on the imaging findings, HCC, an inflammatory pseudotumor of the liver, hepatic adenoma and a hepatic abscess were considered in the differential diagnosis. Within this preoperative differential, we suspected HCC because of the patient's previous history of SVR for hepati- 
Table. Laboratory Data from January, 2012.

\begin{tabular}{lrrlrr}
\hline White blood cells & 4,040 & $/ \mu \mathrm{L}$ & Total protein & 7.1 & $\mathrm{~g} / \mathrm{dL}$ \\
Neutrophils & 58.3 & $\%$ & Albumin & 4.0 & $\mathrm{~g} / \mathrm{dL}$ \\
$\quad$ Basophils & 0.6 & $\%$ & BUN & 17.2 & $\mathrm{mg} / \mathrm{dL}$ \\
\multicolumn{1}{c}{ Eosinophils } & 8.7 & $\%$ & Creatinine & 10.5 & $\mathrm{mg} / \mathrm{dL}$ \\
Lymphocytes & 22.5 & $\%$ & Total Bilirubin & 0.7 & $\mathrm{mg} / \mathrm{dL}$ \\
$\quad$ Monocytes & 7.1 & $\%$ & Direct Bilirubin & 0.1 & $\mathrm{mg} / \mathrm{dL}$ \\
Red blood cells & $471 \times 10^{4}$ & $/ \mathrm{L}$ & AST & 24 & $\mathrm{IU} / \mathrm{L}$ \\
Hemoglobin & 16.2 & $\mathrm{~g} / \mathrm{dL}$ & ALT & 16 & $\mathrm{IU} / \mathrm{L}$ \\
Hematocrit & 46.1 & $\%$ & LDH & 156 & $\mathrm{mg} / \mathrm{dL}$ \\
Platelets & $18.5 \times 10^{4}$ & $/ \mu \mathrm{L}$ & ALP & 369 & $\mathrm{IU} / \mathrm{L}$ \\
PT-INR & 0.99 & & $\gamma$-GTP & 15 & $\mathrm{IU} / \mathrm{L}$ \\
APTT & 33.9 & $\mathrm{sec}$ & $\alpha$-fetoprotein & 2 & $\mathrm{ng} / \mathrm{mL}$ \\
Anti-HCV & $(+)$ & & PIVKA-II & $17 \mathrm{mAU} / \mathrm{mL}$ \\
HCV-RNA & undetectable log $(\mathrm{IU} / \mathrm{mL})$ & &
\end{tabular}

PT-INR: prothrombin time international normalized ratio, APTT: activated partial thromboplastin time, HCV: hepatitis C virus, RNA: ribonucleic acid, BUN: blood urea nitrogen, AST: aspartate aminotransferase, ALT: alanine aminotransferase, LDH: lactate dehydrogenase, ALP: alkaline phosphatase, $\gamma$-GTP: $\gamma$-glutamyltranspeptidase, PIVKA-II: prothrombin induced by vitamin $\mathrm{K}$ absence-II
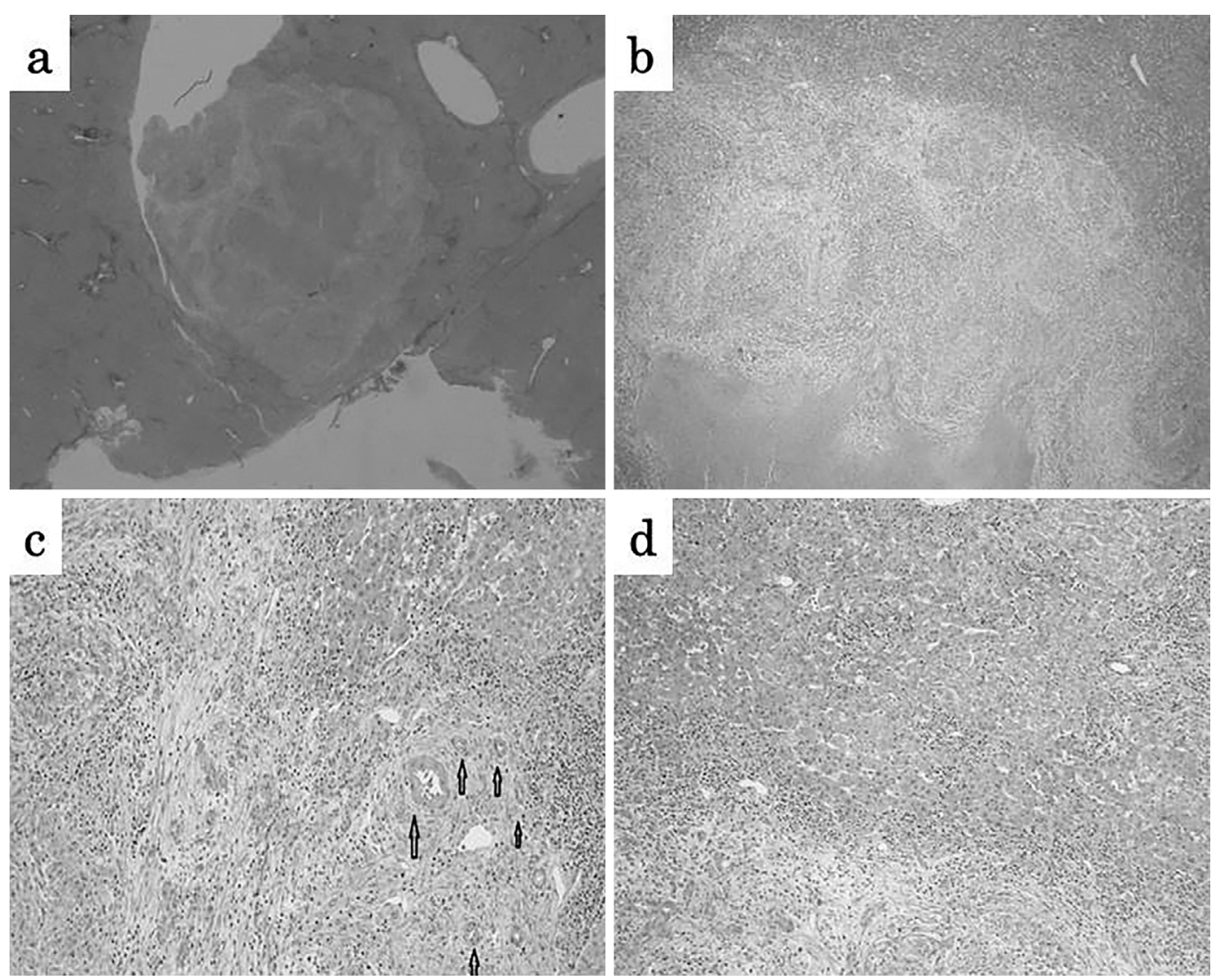

Figure 2. Histopathological examination of LHTB. (a, b) Caseous necrosis surrounded by granulomas, compatible with TB (a: original magnification $\times 5$, b: original magnification $\times 40$ ). (c) Micro-arterial proliferation in the granulomas (arrows) (original magnification $\times \mathbf{1 0 0}$ ). (d) Dilation of the sinusoids with lymphocyte infiltration was detected in the normal hepatic tissue surrounding the hepatic TB lesion (original magnification $\times \mathbf{1 0 0}$ ).

tis $\mathrm{C}$ virus ( $\mathrm{HCV})$, the arterial enhancement on CT/MRI and the low signal intensity on hepatobiliary-phase EOB-MRI. We considered conducting an ultrasonography-guided needle biopsy to confirm the diagnosis; however, we did not perform this procedure due to the $2.7 \%$ risk of needle track seeding following biopsies of HCC lesions (18). After the surgery, we were finally able to accurately diagnose LHTB based on the histopathology.

Recently, a relationship between imaging features and the pathological stage of hepatic TB was reported (7-9). Cao et 
al. reported that there is a relationship between the findings of contrast-enhanced ultrasonography (CEUS) and the pathological features of hepatic TB (8). In summary, during the early stage of granuloma formation, focal sinusoid destruction or congestion leads to increased arterial perfusion and decreased portal perfusion, which present as arterial enhancement and washout during the portal phase. Under conditions of disease progression, the development of granulomas and caseous necrosis results in decreased arterial and portal perfusion and the appearance of hypo- to nonenhancing lesions with an enhancing rim, caused by inflammatory hyperemia in the normal hepatic sinusoids (8). In this case, arterial enhancement of the entire hepatic mass lesion without apparent washout in the portal venous phase was detected on CT (Fig. 1a-c). On EOB-MRI performed one month after the CT scan, a non-enhancing area appeared within the lesion (Fig. 1e). This change was likely due to the progression of hepatic TB and indicates that signs of exudative inflammation were replaced with granulomas and caseous necrosis. The area of caseous necrosis was seen as a small non-enhancing area on the EOB-MRI scan (black zone in Fig. 1g). Micro-arteries also proliferated within the granulomatous tissue (Fig. 2c), which presented as an early enhancing area in the arterial phase and as hypointensity in the hepatobiliary phase (gray zone in Fig. 1g). The sinusoid in the normal hepatic tissue surrounding this lesion was dilated due to lymphocytic infiltration (Fig. 2d), seen as an early enhancing area in the arterial phase and as isointensity in the hepatobiliary phase (white zone in Fig. 1g). To the best of our knowledge, this is the first report of an LHTB patient with imaging changes caused by the progression of TB during the course of the disease. An accurate interpretation of this change might have facilitated our ability to make an accurate preoperative diagnosis of LHTB. It is very important to be aware of LHTB and consider this disease in the differential diagnosis during evaluations of patients with hepatic mass lesions.

ATT is universally effective in cases of LHTB (6). Chong et al. described an algorithm for evaluating patients with suspected hepatobiliary TB (19). According to this algorithm, surgery should be considered if medical or other therapy fails. In the present case, we first suspected that the hepatic mass lesion was HCC; partial hepatectomy was therefore performed, and the correct diagnosis was established postoperatively. Postoperative ATT was not administered because there was no obvious involvement of other organs. No recurrence of TB has been observed since the surgery.

Clinicians should thus be aware of the possibility of LHTB when examining patients with hepatic lesions. In particular, it is necessary to consider LHTB in the differential diagnosis when imaging changes are detected.

The authors state that they have no Conflict of Interest (COI).

\section{References}

1. Yilmaz T, Sever A, Gur S, Killi RM, Elmas N. CT findings of abdominal tuberculosis in 12 patients. Comput Med Imaging Graph 26: 321-325, 2002.

2. Yang ZG, Min PQ, Sone S, et al. Tuberculosis versus lymphomas in the abdominal lymph nodes: evaluation with contrast-enhanced CT. Am J Roentgenol 172: 619-623, 1999.

3. Alvarez SZ, Carpio R. Hepatobiliary tuberculosis. Dig Dis Sci 28: 193-200, 1983.

4. Chien RN, Lin PY, Liaw YF. Hepatic tuberculosis; comparision of miliary and local form. Infection 23: 5-8, 1995.

5. Huang WT, Wang CC, Chen WJ, Cheng YF, Eng HL. The nodular form of hepatic tuberculosis: a review with five additional new cases. J Clin Pathol 56: 835-839, 2003.

6. Amarapurkar DN, Patel ND, Amarapurkar AD. Hepatobiliary tuberculosis in western India. Indian J Patholo Microbiol 51: 175181, 2008.

7. Yu RS, Zhang SZ, Wu JJ, Li RF. Imaging diagnosis of 12 patients with hepatic tuberculosis. World J Gastroenterol 10: 1639-1642, 2004.

8. Cao BS, Li XL, Li N, Wang ZY. The nodular form of hepatic tuberculosis: contrast-enhanced ultrasonographic findings with pathologic correlation. J Ultrasound Med 29: 881-888, 2010.

9. Nizar M, Zeineb M, Sadri BA, et al. Pseudo-tumoral hepatic tuberculosis discovered after surgical resection. Clin Pract 2: e27, 2012.

10. Alvarez SZ. Hepatobiliary tuberculosis. J Gastroenterol Hepatol 13: 833-839, 1998.

11. Hussain W, Mutimer D, Harrison R, Hubscher S, Neuberger J. Fulminant hepatic failure caused by tuberculosis. Gut 36: 792-794, 1995.

12. Spiegel CT, Tuazon CU. Tuberculous liver abscess. Tubercle $\mathbf{6 5}$ : 127-131, 1984.

13. Hulnick DH, Megibow AJ, Naidch DP, Hilton S, Cho KC, Balthazar EJ. Abdominal tuberculosis: CT evaluation. Radiology 157: 199-204, 1985.

14. Oliva A, Duarte B, Jonasson O, Nadimpalli V. The nodular form of local hepatic tuberculosis. A review. J Clin Gastroenterol 12: 166-173, 1990.

15. Dias LT, Rodrigues GC, Barbosa DS, Lacerda DC, Ruas MF. Primary nodular hepatic tuberculosis mimicking hepatic neoplasia in an immunocompetent host. Braz J Infect Dis 13: 153-154, 2009.

16. Levine C. Primary macronodular hepatic tuberculosis: US and CT appearances. Gastrointest Radiol 15: 307-309, 1990.

17. Sheen-Chen SM, Chen MC, Hu TH, et al. Computed tomography and angiography in hepatic tuberculosis mimicking liver tumor. Int J Tuberc Lung Dis 5: 876-878, 2001.

18. Silva MA, Hegab B, Hyde C, Guo B, Buckels JA, Mirza DF. Needle track seeding following biopsy of liver lesions in the diagnosis of hepatocellular cancer: a systematic review and meta-analysis. Gut 57: 1592-1596, 2008.

19. Chong VH, Lim KS. Hepatobiliary tuberculosis. Singapore Med J 51: 744-751, 2010.

(C) 2016 The Japanese Society of Internal Medicine http://www.naika.or.jp/imonline/index.html 